Claremont Colleges

Scholarship@ Claremont

Pitzer Faculty Publications and Research

Pitzer Faculty Scholarship

7-20-2012

\title{
Homology in Developmental Psychology
}

David S. Moore

Pitzer College

\section{Recommended Citation}

Moore, D. S. and Moore, C. (2013), Homology in developmental psychology. Dev. Psychobiol., 55: 1-3. doi: 10.1002/dev.21062

This Article - preprint is brought to you for free and open access by the Pitzer Faculty Scholarship at Scholarship @ Claremont. It has been accepted for inclusion in Pitzer Faculty Publications and Research by an authorized administrator of Scholarship @ Claremont. For more information, please contact scholarship@cuc.claremont.edu. 
Running head: Homology and Development

The content of this open-access pre-print article is the same as that contained in the published article with the following reference:

Moore, D. S. \& Moore, C. (Eds.). (2013). Homology in developmental psychology. Developmental Psychobiology, 55, 1-3.

\section{Editorial}

Homology in Developmental Psychology

David S. Moore*

Pitzer College and

Claremont Graduate University
Chris Moore*

Dalhousie University

May 29, 2012

*Both authors contributed equally to this work 


\section{Editorial}

Homology in Developmental Psychology

This special issue is the result of an NSF-funded workshop held in the summer of 2011 at Dalhousie University in Halifax, Nova Scotia. The goal of the workshop was to explore whether the biological concept of homology, so powerful in the understanding of evolution, could help with our understanding of developmental psychology. The basic rationale is simple. Like evolution, development entails change associated with time allied with continuity across time. The application of the homology concept in biology allows for the identification of continuity across species that is due to evolution from a common ancestor. And in tracing phylogenetic change over time, the homology concept allows the identification of sameness and therefore of relatedness over time. The goal of developmental psychology is to understand how psychological and behavioral characteristics at any point in the lifespan emerge from characteristics present earlier in development. A comprehensive developmental psychology also requires identifying both sameness and difference in characteristics over time. Thus, evolutionary change and developmental change can be understood as being analogous in some respects. But this superficially simple analogy between phylogeny and ontogeny hides a range of complications, several of which are considered in this collection of papers.

In exploring the potential value of importing the homology concept from evolutionary biology into developmental psychology, participants in the workshop effectively had to address two questions. The first was whether application of the homology concept to ontogeny might yield valuable insights, like the insights that emerged by applying this concept to phylogeny. Because biologists have already had some success applying the homology concept to development (Bertossa, 2011; Wagner, 2007), we had reason to anticipate an affirmative answer 
to this question. In contrast, the second question was whether the homology concept can be applied successfully to psychological characteristics. Because behavioral, affective, and cognitive processes are less obviously amenable to homological analysis than are anatomical features (Atz, 1970), an affirmative answer to this question was not assured.

To examine whether there is potential for the extension of the concept of homology to developmental psychology, the workshop brought together an interdisciplinary group of scholars. Biologists, comparative psychologists, developmental psychologists, neuroscientists, and philosophers of biology and psychology, convened for two days of talks and discussion. The contributors all circulated drafts of their papers ahead of the meeting and then presented their ideas to the group. The outcome of their ideas, refined through discussion and debate, is the series of papers in this special issue.

The papers begin with an exposition of the concept of homology as it has been used in biology, both historically and in the present day. In his paper, Brian Hall traces the origins of the homology concept and shows how it has progressed within biology. David Moore picks up from there and considers the potential utility of the concept for developmental psychologists. In particular, he argues that homology thinking may assist with resolving whether developmentally early-emerging and developmentally late-emerging characteristics should be considered versions of the same trait. Together, these papers provide a more detailed rationale for the belief that the homology concept might prove useful to developmental psychologists.

The papers by Paul Griffiths, Frances Champagne, and Michael Anderson \& Marcie Penner-Wilger provide valuable interdisciplinary perspectives in that they address levels of analysis that are not strictly psychological. Approaching the issue of homology in developmental science from a philosophical point of view, Griffiths considers problems that could arise when 
modes of explanation favored by evolutionary biologists are recruited to explain development. Although Griffiths is optimistic that the homology concept might be usefully imported into developmental psychology, his paper provides an important warning about the potential dangers of conflating evolutionary and developmental explanations. In Champagne's paper, we find a discussion of how epigenetic mechanisms influence gene expression; these mechanisms, she argues, allow for a process homology underlying environmental effects on development (i.e., phenotypic plasticity) across species. Anderson and Penner-Wilger consider the idea of developmental homology at a higher level of analysis, beginning with a presentation of compelling data that indicate that neural circuits serve multiple behavioral purposes. These authors then argue that thinking about the reuse of neural circuits in terms of developmental homology might be a promising strategy that could help explain diverse phenomena, including why certain cognitive domains have some of the specific features they do, why some of these kinds of features re-appear in multiple task contexts, and why critical periods (and other order effects in learning) might manifest as they do.

The remaining papers focus on particular psychological and behavioral phenomena and analyze them through the lens of homology. Thomas Suddendorf and his colleagues address the somewhat controversial phenomenon of neonatal imitation and its relation to later forms of imitation. They suggest that an explicit treatment in terms of homology clarifies what is at stake and they point to methodological approaches required to assess whether indeed it is appropriate to consider neonatal imitation as a behavioral homologue of later imitation. Chris Moore suggests that the identification of behavioral structure can help us recognize continuity in psychological function that might not be obvious from surface forms. He uses this approach to argue that triadic social interactive structures in infancy are developmentally homologous with 
later emerging structures in language. Jason Clark analyzes human emotions in the context of developmental homology. He argues that there are important connections among the emotions that emerge at different stages of development, including persistence across development, and that later emerging emotions may be understood as serial homologues of earlier emerging ones. Robert Lickliter and Lorraine Bahrick consider an aspect of perceptual processing and selective attention. They review some of their empirical work with infants and children that shows how redundancy in information presented intermodally privileges attention to, and processing of, amodal properties. They show how such processing holds across development and consider whether it may be fruitfully construed as an example of developmental homology. And George Michel reviews his work on handedness against the background of homology, arguing that whereas handedness shows evidence of emerging through a dynamic system and of being developmentally continuous, it is probably a mistake to interpret this continuity as a sign of homology.

Finally, Mark Blumberg's article uses empirical data on the early development of sleep to examine if there might be value in considering complex behaviors present at two different points in development to be developmentally homologous. He concludes that conceptualizing development in this way is unlikely to foster new insights, even as he remains sanguine about the possibility of developmentalists continuing to exploit more traditional understandings of homology (i.e., cross-species similarities resulting from common descent). Blumberg's cogent critique notwithstanding, we continue to believe there is something potentially important about the fact that behaviors at two points in time might serve the same function in different ways and therefore not be homologous (e.g., feeding and suckling, as discussed by Hall \& Williams, 1983), but that other behaviors at two points in time might serve the same function in the same 
ways, for reasons having to do with the fact that one of them is a developmental precursor of the other; the latter behaviors might well be thought of as homologous. Of course, Blumberg's analysis strikes an important cautionary note: conceptual tools developed in one discipline can be used in different disciplines in a way that confuses more than it clarifies, so additional deliberation regarding the wisdom of thinking in terms of developmental homology is warranted. But taken together, we believe this collection of papers represents an intriguing and promising initial consideration of some of the potential benefits and pitfalls of approaching the questions of developmental psychology using the homology concept. 


\section{References}

Atz, J. W. (1970). The application of the idea of homology to behavior. In L. R. Aronson, E. Tobach, D. S. Lehrman, \& J. S. Rosenblatt (Eds.), Development and evolution of behavior: Essays in memory of T. C. Schneirla (pp. 53-74). San Francisco: Freeman.

Bertossa, R. C. (2011). Morphology and behaviour: Functional links in development and evolution. Philosophical Transactions of the Royal Society of London (B), 366, 2056-2068.

Hall, W. G., \& Williams, C. L. (1983). Suckling isn't feeding, or is it? A search for developmental constraints. Advances in the Study of Behavior, 13, 219-254.

Wagner, G. P. (2007). The developmental genetics of homology. Nature Reviews Genetics, 8, 473479. 
Author Note

Chris Moore*

Department of Psychology, 1355 Oxford Street,

Dalhousie University, Halifax, NS, B3H 4J1 CANADA

E-mail address: moorec@dal.ca

David S. Moore*

Pitzer College, 1050 N. Mills Avenue, Claremont, CA 91711, USA

E-mail address: dmoore@pitzer.edu

*Both authors contributed equally to this work 